\title{
(2) OPEN ACCESS \\ Allergic diseases influence symptom severity and T lymphocyte subgroups of children with tic disorders
}

\author{
Xiumei Liu (i) , ${ }^{1}$ Xueming Wang, ${ }^{1}$ Xiaoling Zhang, ${ }^{2}$ Ai hua $\mathrm{CaO}^{3}$
}

\begin{abstract}
'Child Care Center, Fujian Provincial Maternity and Children's Hospital, Fuzhou, China

${ }^{2}$ Department of Pediatrics, Weifang Medical School, Weifang, China

${ }^{3}$ Department of Pediatrics, Shandong University Qilu Hospital, Jinan, Shandong, China
\end{abstract}

\section{Correspondence to} Dr Ai hua Cao, Department of Pediatrics, Shandong University Qilu Hospital, Jinan, Shandong, China; xinercah@163.com

$\mathrm{XL}$ and $\mathrm{XW}$ are joint first authors.

Accepted 22 June 2021

\section{Check for updates}

(c) American Federation for Medical Research 2021. Re-use permitted under CC BY-NC. No commercial re-use. Published by BMJ.

\begin{tabular}{l}
\hline To cite: Liu X, Wang X, \\
Zhang X, et al. \\
J Investig Med Epub \\
ahead of print: [please \\
include Day Month Year]. \\
doi:10.1136/jim-2021- \\
001788
\end{tabular}

\begin{abstract}
Tic disorders (TD) are childhood-onset neurological disorders. Immune system dysregulation has been postulated to play a role in TD, and its mechanisms likely involve dysfunctional neural-immune crosstalk, which ultimately leads to altered maturation of the brain pathways that control different TD clinical manifestations and behavioral and emotional damages. Clinical studies have demonstrated an association between TD and allergies and overactive immune responses at a systemic level. In this study, the Yale Global Tic Severity Scale was taken as a global measure of tic severity. Compared with the control group, the group of children with TD plus allergic diseases displayed significantly increased Yale total scores $(p<0.05)$, which suggests that children with TD plus allergic diseases have heavier tic symptoms. Both motor and vocal tic scores are higher in the group of children with TD plus allergy compared with the control group. We counted immune cell subpopulations using FACS. T lymphocyte subset comparison of CD3, CD4, CD8, and $C D 4: C D 8$ expression ratios revealed that the level of CD3, CD4, and CD4:CD8 in children with TD plus allergic diseases was significantly lower than those of children with TD without allergic diseases. These differences were statistically significant $(p<0.05)$ and suggest that children with TD plus allergic diseases have imbalanced T lymphocyte subsets. We concluded that allergy increased the severity of TD through an imbalance in cellular immunity. Studies need to be done to show whether treatment of allergic symptoms leads to a decrease in TD manifestations.
\end{abstract}

\section{INTRODUCTION}

Tic disorders (TD) are common neurodevelopmental disorders in childhood characterized by various forms of motor and vocal tics. Severity of tic rises and falls, with the peak generally occurring in early adolescence. ${ }^{1}$ The prevalence of TD has risen in recent years, causing pain and interfering with the daily life of patients. ${ }^{2}$ In addition to the core symptoms of TD, affected individuals often present with behavioral difficulties, such as comorbid obsessive-compulsive disorder (OCD), attention deficit-hyperactivity disorder, etc. ${ }^{3}$ In a subset of children with TD, symptoms continue to adolescence and

\section{Significance of this study}

What is already known about this subject?

- Tic disorders (TD) are common neurodevelopmental disorders in childhood.

- The relationship between TD and allergic diseases has gained attention among scientists.

- The association between allergy parameters and TD has not been clearly demonstrated to date.

What are the new findings?

- We investigate symptom severity and T lymphocyte subgroups in children with TD plus allergic diseases.

- Allergic disease increases illness severity in children with TD.

- Allergic disease aggravates imbalance of T lymphocyte subsets and cellular immune dysfunction in children with TD.

How might these results change the focus of research or clinical practice?

- The involvement of the immune system in TD is a relatively recent area of investigation.

- Allergic disease should be prevented and treated actively upon clinical diagnosis and treatment of TD.

adulthood and seriously affect their quality of life. ${ }^{4}$ The etiology of TD is currently not known and the interplay between genetic, environmental, hormonal, and immunological factors might be relevant. ${ }^{5}$

There has been growing interest in the association between immune dysfunction and TD. Immune system problems associated with TD have mostly been reported in studies investigating pediatric autoimmune neuropsychiatric disorders associated with streptococcal infections (PANDAS). ${ }^{6}$ The PANDAS hypothesis indicates that immune mechanisms are involved in TD, and interactions between the immune system and the nervous system have been implicated in the occurrence of TD. ${ }^{7}$ In a previous study, the proponents of PANDAS pointed out that elevated antistreptolysin $\mathrm{O}$ titers were described in children with TD compared 
with those in controls. ${ }^{8}$ Another study suggested a significant correlation between streptococcal-associated tics and elevated anti-dopamine receptor D1 and antilysoganglioside antineuronal antibodies in serum. ${ }^{9}$ Youth and young adults with chronic TD may have underlying infectious/ immunological etiology. In addition to PANDAS, previous studies have reported functional differences in the immune system of children and adolescents diagnosed with TD in association with Enterovirus infections. ${ }^{10}$ Supporting the conclusion that patients with TD have immune system problems, some patients in various studies did not respond to antipsychotics, but did respond to adrenocorticotropic hormone, prednisolone, and/or plasmapheresis. ${ }^{11} 12$

Allergy is a hypersensitivity reaction initiated by specific immunological mechanisms. Allergic disease has become a major burden in the modern society of China due to a recent rise in its prevalence. Recent studies have reported that children with TD often present eczema, allergic rhinitis, allergic conjunctivitis, asthma, or other allergic diseases. ${ }^{13-15}$ Therefore, the relationship between TD and allergic diseases has gained attention among scientists and requires further study. Analysis of the lymphocyte subpopulation in TD suggests a possible systemic activation of several $\mathrm{T}$ cell and $\mathrm{B}$ cell subtypes. Genes related to both cell and antibody-mediated immune responses may be overexpressed at specific ages in youngsters with TD. A significant positive correlation between Tourette syndrome (TS) and allergy has been reported. ${ }^{16}$ To date, the association between allergy parameters and TD has not been clearly demonstrated. Here, we investigate symptom severity and $\mathrm{T}$ lymphocyte subgroups in children with TD plus allergic diseases.

\section{PATIENTS AND METHODS \\ Subjects}

We collected data of children with TD from the Children's Healthcare Outpatient Clinic in Yantai Yuhuangding Hospital from January 2015 to January 2017. Two groups of subjects were established for this study: (1) subjects with TD and (2) subjects with TD plus allergic diseases. The TD cohort consisted of 135 children (98 boys and 37 girls) aged 6-9 years who met the The Diagnostic and Statistical Manual of Mental Disorders, Fifth Edition (DSM-V) criteria for TD. Eighty-five cases of children with TD plus chronic allergic diseases were recruited (53 cases of allergic rhinitis, 11 cases of atopic dermatitis, and 21 cases of asthma) at random, and this patient group included 65 boys and 20 girls aged 6-10 years. The DSM-V diagnostic criteria for TD had to be fulfilled and a diagnosis of allergy was made by a pediatric allergy specialist. Exclusion criteria were habitual convulsions, myoclonic epilepsy, rheumatoid chorea, liver degeneration, traumatic brain injuries, encephalitis sequela, and other extrapyramidal diseases. Subjects taking allergy medication(s) were also excluded.Written informed consent was obtained from the parents or guardians of the subjects.

\section{Yale Global Tic Severity Scale}

The Yale Global Tic Severity Scale (YGTSS) is an index of tic severity that is commonly used for children and adolescents. ${ }^{17}$ This index consists of a semistructured, clinicianrated interview designed to provide a global measure of tic severity over a previous period of 7-10 days. Tic severity is determined based on evaluation of number, frequency, intensity, complexity, and interference of motor and phonic tics and a global impairment rating. Each dimension is scored on a 5-point scale, separately for vocal and motor tics. A total severity score is obtained by summing all scores across vocal and motor tics (range $0-50$ ) or separately for vocal and motor tics. Severity of disease was classified into three grades according to the total score on the YGTSS: mild TD: $10-19$ points; moderate TD: $20-39$ points; severe TD: 40-50 points. The instrument has demonstrated good internal consistency and convergent and divergent validity. ${ }^{18}$

\section{T lymphocyte subgroup detection}

Detection of $\mathrm{T}$ lymphocyte subgroups was performed on children of the research group and the control group. The two groups of children were required to take $2 \mathrm{~mL}$ of venous blood in the morning after 8 hours of fasting. The blood sample was treated with $1 \%$ in 0.1 units of heparin in vitro and slightly shaken up and down, after which the samples were immediately sent to the laboratory for testing. Samples were centrifuged for $15 \mathrm{~min}$, and stained with fluorescein isothiocyanate-conjugated or phycoerythrin-conjugated antimarker monoclonal antibodies in $100 \mathrm{~mL}$ phosphate buffered solution for 6 hours, as suggested by the manufacturer. Samples were then analyzed using flow cytometry to determine the total percentage of $\mathrm{T}$ lymphocytes (CD3 ratio), percentage of $\mathrm{T}$ helper lymphocytes (CD4 ratio), and $\mathrm{T}$ lymphocyte percentage (CD8 and $\mathrm{CD} 4: \mathrm{CD} 8$ ratios) (FACS caliber, BD, USA). After the blood samples were analyzed, the required data were recorded.

\section{Statistical analysis}

Variable distributions were examined for normality, and non-parametric statistics were used in cases where scores were not normally distributed. We completed statistical analysis according to the type of statistical data for hypothesis testing. Homogeneity test of variance was completed with $F$ test. Statistical differences between groups were assessed using different methods. Parts of values are reported as mean $\pm \mathrm{SD}$. Comparisons of $\mathrm{T}$ lymphocyte subsets and YGTSS scores between the two cohorts (TD and TD plus allergic diseases) were performed using Student's t-test. Pearson's $\chi^{2}$ test was used to detect the constituent ratio of the two groups. SPSS V.15.0 was used for all statistical calculations. All tests were two-sided and a p value $<0.05$ was considered statistically significant.

\section{RESULTS}

\section{Demographic features and clinical characteristics of the two groups}

Two groups of subjects were established for this study: subjects with TD and subjects with TD plus allergic diseases. The demographic features and clinical characteristics of the two groups are shown in table 1 . In our study, the mean age of children diagnosed with TD $(n=135)$ was $7.2 \pm 1.5$ years, and the mean age of the 85 subjects with TD plus allergic diseases was $7.6 \pm 1.6$ years (table 1 ). There were no statistically significant differences between the two groups in terms of age and sex $(p>0.05)$. The TD sample was further divided into three subgroups: transient TD, chronic 
Table 1 Demographic features of subjects with TD and subjects with TD plus allergic diseases

\begin{tabular}{|c|c|c|c|c|}
\hline Variables & TD $(n=135)$ & $\begin{array}{l}\text { TD plus allergy } \\
(\mathrm{n}=85)\end{array}$ & $t\left(\chi^{2}\right)$ & $P$ value \\
\hline \multicolumn{5}{|l|}{ Sex } \\
\hline Male & 98 & 60 & $0.1035^{*}$ & 0.7476 \\
\hline Female & 37 & 25 & & \\
\hline Age & $7.2 \pm 1.5$ & $7.6 \pm 1.6$ & $1.8767 \dagger$ & 0.0619 \\
\hline \multicolumn{5}{|l|}{ Type of TD } \\
\hline TTD & 58 & 27 & $2.9433^{*}$ & 0.2296 \\
\hline CTD & 37 & 30 & & \\
\hline TS & 40 & 28 & & \\
\hline \multicolumn{5}{|l|}{ Severity } \\
\hline Mild TD & 80 & 35 & $7.8004^{*}$ & 0.0202 \\
\hline Moderate TD & 42 & 34 & & \\
\hline Severe TD & 13 & 16 & & \\
\hline
\end{tabular}

*Pearson's $\chi^{2}$ test.

tt-test.

CTD, chronic tic disorder; TD, tic disorder; TS, Tourette syndrome; TTD, transient tic disorder.

TD, and TS. The proportion of chronic TD and TS in the research group, TD plus allergic diseases, was higher than in the control group (table 1).

\section{Comparison of tic severity between children with and without allergic diseases}

In this study, the YGTSS was taken as a global measure of tic severity. Tic severity was determined based on the evaluation of number, frequency, intensity, complexity, and interference of motor and vocal tics and a global impairment rating. Compared with the control group, the group of children with TD plus allergic diseases displayed significantly increased Yale scores $(p<0.05)$, which suggests that children with TD plus allergic diseases have heavier tic symptoms (table 2). Both motor and vocal tic scores were higher in the group of children with TD plus allergy compared with the control group. Furthermore, there were more moderate and severe tics in children with TD plus allergic diseases.

\section{Comparison of T lymphocyte subsets between the two cohorts}

General immune response features of an individual at a definite time point can be determined by the quantitative distribution of various immune cell subpopulations in the peripheral blood. Here, we counted immune cell subpopulations using FACS. In our study, T lymphocyte subset comparison of $\mathrm{CD} 3, \mathrm{CD} 4, \mathrm{CD} 8$, and CD4:CD8 expression

Table 2 YGTSS assessment in children with TD with or without allergic disease

\begin{tabular}{lllll}
\hline YGTSS score & TD $(\mathbf{n}=135)$ & $\begin{array}{l}\text { TD plus allergy } \\
(\mathbf{n}=85)\end{array}$ & $\mathbf{t}\left(\mathbf{X}^{2}\right)^{*}$ & $\mathbf{P}$ value \\
\hline Motor tics & $24.5 \pm 3.7$ & $20.2 \pm 3.2$ & 9.13 & 0.0000 \\
Vocal tics & $10.4 \pm 4.1$ & $8.5 \pm 3.8$ & 3.502 & 0.0006 \\
Total score & $34.9 \pm 6.5$ & $28.7 \pm 6.1$ & 7.156 & 0.0000 \\
\hline
\end{tabular}

*Pearson's $\chi^{2}$ test.

TD, tic disorder; YGTSS, Yale Global Tic Severity Scale.
Table 3 T lymphocyte subset test results for the two cohorts of children

\begin{tabular}{lllcc}
\hline $\begin{array}{l}\text { T lymphocyte } \\
\text { subset }\end{array}$ & TD $(\mathbf{n}=135)$ & $\begin{array}{l}\text { TD plus allergy } \\
(\mathbf{n}=\mathbf{8 5})\end{array}$ & $\mathbf{t}\left(\mathbf{t}^{\prime *}\right)$ & P value \\
\hline CD3 & $62.1 \pm 8.7$ & $65.4 \pm 7.8$ & 2.8492 & 0.0048 \\
CD4 & $28.3 \pm 5.1$ & $38.3 \pm 5.1$ & 14.161 & 0.0000 \\
CD8 & $31.5 \pm 6.2$ & $30.7 \pm 6.5$ & 0.9146 & 0.3614 \\
CD4:CD8 & $0.8 \pm 0.1$ & $1.3 \pm 0.3$ & $-14.855^{*}$ & $<0.05$ \\
\hline
\end{tabular}

* $t$ 'test was used when the variance of two samples is not equal. TD, tic disorder.

ratios revealed that the level of $\mathrm{CD} 3, \mathrm{CD} 4$, and $\mathrm{CD} 4: \mathrm{CD} 8$ in children with TD plus allergic diseases was significantly lower than those of children with TD without allergic diseases. These differences were statistically significant $(\mathrm{p}<0.05)$ and suggest that children with TD plus allergic diseases have imbalanced T lymphocyte subsets (table 3).

\section{DISCUSSION}

\section{Association between allergy and TD}

Allergy is a hypersensitivity reaction initiated by specific immunological mechanisms. Allergic disease refers to an allergic reaction mediated by IgE, which leads to a variety of dysfunctions or tissue damages, such as bronchial asthma, allergic rhinitis, and allergic dermatosis. ${ }^{19}$ Allergic diseases have become a major burden in China due to a recent rise in prevalence. The etiology of allergic disease is complex, including genetic factors, allergens, various physical and chemical factors, and mental emotions, which is similar to the etiology of TD. ${ }^{20}$ To date, few studies have explored the association between allergy and TD. ${ }^{21}$ Using the Taiwan National Health Insurance Research Database from 1996 to 2010, Chen and coworkers ${ }^{22}$ conducted a retrospective study and found a significant association between TD and allergic diseases. These authors found a higher risk of allergic rhinitis, asthma, dermatitis, and conjunctivitis in patients with TS; however, there were no more details on the possible susceptibility of patients with TS to allergic mechanisms. ${ }^{22}$ A preliminary study carried out in Turkey found associations between allergic diseases and TS and/or OCD and discovered different associations between various allergic diseases (rhinitis or eczema) and neuropsychiatric disorders (TD or OCD). ${ }^{16}$

Recently, we have found that children with TD seek help at outpatient clinics for complaints such as blinking of the eyes, wrinkling of the nose, and hawking (clearing the throat). Many children with TD are often diagnosed with diseases such as allergic conjunctivitis, allergic rhinitis, and allergic pharyngitis when they see a doctor. The occurrence of TD also has certain seasonal characteristics, with the incidence of TD being very high during spring and autumn, which coincides with the peak times for multiple chronic allergic diseases. Furthermore, the parents of children with TD have reported that tic symptoms increase after their children have ingested certain allergens in food. ${ }^{23}$ All of this evidence supports a relationship between TD and allergic factors, and the risk increased with number of allergic comorbidities and with age. ${ }^{24} 25$

Another study reported that patients with TS who also suffer tics that resemble asthma symptoms were often 
diagnosed with asthma and received asthma treatments. ${ }^{26}$ On the other hand, asthma is reported to be associated with several psychiatric disorders including TD. ${ }^{27}$ Many children with TS are incorrectly diagnosed and treated for asthma or rhinitis. It is recommend that clinicians who evaluate allergies in children take TD into account. ${ }^{28}$ Therefore, it is necessary to obtain detailed medical history and physical examination, including mental assessments and necessary auxiliary examinations, before diagnosing TD. The purpose of such an inspection is to rule out other diseases, especially allergic disease.

\section{Immunity and neurodevelopmental disorders}

The association between immunity and neurodevelopmental disorders has been extensively investigated in autism, and the potential involvement of cellular and humoral immunities has been suggested to play a role in establishing synaptic connectivity during development. ${ }^{29}$ A similar link has also been proposed for TD, where the interplay between genetic, environmental, hormonal, and immunological factors might be relevant. Previous research has shown that some children with TD display immune injuries. Genetic susceptibility and immune damage play important roles in the occurrence and development of TD, dysregulation of the nerve, endocrine, and immune systems, and induction of tic symptoms. ${ }^{30}$ With analogy to autism, genetic and early life environmental factors could foster the involvement of immune mechanisms in the abnormal developmental trajectories postulated in TS, as well as lead to systemic immune dysregulation in this condition. ${ }^{31}$ Immune system problems associated with TD have mostly been reported in studies investigating PANDAS. Streptococcal infection in patients with TS is associated with immune and cytokine dysfunctions, which can be potentially managed with immunomodulator therapy. ${ }^{32} 33$

We evaluated the illness severity of two groups of children with TD with and without allergic diseases. Our results show that children with TD with allergic diseases have higher motor tic score, vocal tic score, and total scores than those without allergic diseases. Allergic disease is related to tic symptoms and severity of TD. Furthermore allergic disease increases clinical symptoms and emotion and social communication problems in patients with TD. Allergy-associated immune dysfunction worsens tics and other abnormal behaviors and contributes to their pathogenesis. ${ }^{34}$ However, there is no clear evidence at present to support this complex neural-immune interplay in TD.

\section{T lymphocyte subsets in children with TD with allergy}

$\mathrm{T}$ cell-mediated cellular immunity plays an important role in body protection. The subsets of $\mathrm{T}$ cells are mutually regulated and balanced in a steady state. When homeostasis is destroyed, the body will show some pathological phenomenon.

In this study, we found that the level of $\mathrm{T}$ cells in children with TD with allergic diseases is lower than in children with TD without allergic diseases. CD3 + T cells, CD4 + T cells, and CD4:CD8 expression levels are lower in children with TD with allergic diseases compared with the control group. Allergic disease aggravates imbalance of $\mathrm{T}$ lymphocyte subsets and cellular immune dysfunction in children with TD. Analysis of the lymphocyte subpopulation in TS suggests a possible systemic activation of several $\mathrm{T}$ cell and $B$ cell subtypes, whereas the observed decreased number of $\mathrm{T}$ regulatory lymphocytes might predispose to autoimmunity. ${ }^{35}$ Lymphocytes may also be modulated by other neurotransmitters, including glutamate, acetylcholine, and serotonin, which are important neurotransmitters of the nervous system critically needed for the brain's development and function. These neurotransmitters might also be involved in the pathophysiology of TD in general. ${ }^{36}$

Specific cytokines are involved in the communication between several immune cells, and these can change neuroimmunological functions and behavior via transporting signals to the central nervous system. Cytokines secreted by CD4+ T cells are very important mediators in human's immune system. The receptors of these cytokines are found in various systems, including the nervous system. When there are abnormal $\mathrm{T}$ cells and cytokines, associated neurological diseases appear. Other neurological and psychiatric conditions associated with dysfunction of the dopaminergic circuits, such as Parkinson's disease and schizophrenia, also present with altered immune function. Dopamine modulates the immune cells in an autocrine or paracrine fashion, and lymphocytes express dopamine receptors on their surface.

To summarize, the involvement of the immune system in TD is a relatively recent area of investigation. Allergic diseases increase illness severity and cellular immune function in children with TD. Allergic diseases should be prevented and treated actively upon clinical diagnosis and treatment of TD. As a final question for future research, it is interesting to hypothesize whether the altered immune cell function might contribute to abnormal brain maturation, and consequently facilitate the manifestation of tics and associated dysfunctional behaviors. In the future, more investigations are needed across larger clinical samples in order to confirm the characterization of $\mathrm{T}$ lymphocyte subgroups in patients with TD. Furthermore, specific cytokines are involved in the communication between immune cells, which can change neuroimmunological functions and behavior via transporting signals to the central nervous system. Additional research is needed in this area.

Contributors XL: methodology, software, investigation, writing-original draft. XW: methodology, formal analysis, software, writing-original draft. XZ: investigation, formal analysis. AC: conceptualization, resources, project administration, funding acquisition. All authors have read and agreed to the published version of the manuscript.

Funding This study was funded by the National Natural Science Foundation of China (no. 81871076) and Shandong Natural Science Foundation (ZR2017LH038).

Competing interests None declared.

Patient consent for publication Not required.

Ethics approval The study was approved by the Ethics Committee of Yantai Yuhuangding Hospital.

Provenance and peer review Not commissioned; externally peer reviewed. Data availability statement The data used to support the findings of this study are available from the corresponding author on reasonable request.

Open access This is an open access article distributed in accordance with the Creative Commons Attribution Non Commercial (CC BY-NC 4.0) license, which permits others to distribute, remix, adapt, build upon this work noncommercially, and license their derivative works on different terms, provided the original work is properly cited, an indication of whether changes were 
made, and the use is non-commercial. See: http://creativecommons.org/ licenses/by-nc/4.0/.

\section{ORCID iD}

Xiumei Liu http://orcid.org/0000-0002-9119-7827

\section{REFERENCES}

1 Hartmann A, Worbe Y. Tourette syndrome: clinical spectrum, mechanisms and personalized treatments. Curr Opin Neurol 2018;31:504-9.

2 Dale RC. Tics and Tourette: a clinical, pathophysiological and etiological review. Curr Opin Pediatr 2017;29:665-73.

3 Cavanna AE. Gilles de la Tourette syndrome as a paradigmatic neuropsychiatric disorder. CNS Spectr 2018;23:213-8.

4 Groth C, Skov L, Lange T, et al. Predictors of the clinical course of Tourette syndrome: a longitudinal study. J Child Neurol 2019;34:913-21.

5 Yael D, Vinner E, Bar-Gad I. Pathophysiology of tic disorders. Mov Disord 2015;30:1171-8.

6 Gilbert DL. Inflammation in tic disorders and obsessive-compulsive disorder: are pans and PANDAS a path forward? J Child Neurol 2019;34:598-611.

7 Pranzatelli MR, Tate ED, Allison TJ. Case-control, exploratory study of cerebrospinal fluid chemokines/cytokines and lymphocyte subsets in childhood Tourette syndrome with positive streptococcal markers. Cytokine 2017;96:49-53

8 Lepri G, Rigante D, Bellando Randone S, et al. Clinical-Serological characterization and treatment outcome of a large cohort of Italian children with pediatric autoimmune neuropsychiatric disorder associated with streptococcal infection and pediatric acute neuropsychiatric syndrome. J Child Adolesc Psychopharmacol 2019:29:608-14.

9 Pozzi M, Pellegrino P, Carnovale C, et al. On the connection between autoimmunity, tic disorders and obsessive-compulsive disorders: a meta-analysis on anti-streptolysin 0 titres. J Neuroimmune Pharmacol 2014;9:606-14.

10 Tsai C-S, Yang Y-H, Huang K-Y, et al. Association of tic disorders and enterovirus infection: a nationwide population-based study. Medicine 2016;95:e3347.c.

11 Cox CJ, Zuccolo AJ, Edwards EV, et al. Antineuronal antibodies in a heterogeneous group of youth and young adults with tics and obsessivecompulsive disorder. J Child Adolesc Psychopharmacol 2015;25:76-85.

12 Nave $\mathrm{AH}$, Harmel P, Buchert R, et al. Altered cerebral glucose metabolism normalized in a patient with a pediatric autoimmune neuropsychiatric disorder after streptococcal infection (PANDAS)-like condition following treatment with plasmapheresis: a case report. BMC Neurol 2018;18:60.

13 Chen L, Chen X, Ke N, et al. Association between allergic conjunctivitis and provisional tic disorder in children. Int Ophthalmol 2020;40:247-53.

14 Zhou H, Chen Z, Zhao W, et al. Evaluation of neuropsychiatric comorbidities and their clinical characteristics in Chinese children with asthma using the mini kid tool. BMC Pediatr 2019;19:454.

15 Catal F, Topal E, Soylu N, et al. Psychiatric disorders and symptoms severity in preschool children with atopic eczema. Allergol Immunopathol 2016:44:120-4.

16 Yuce M, Guner SN, Karabekiroglu K, et al. Association of Tourette syndrome and obsessive-compulsive disorder with allergic diseases in children and adolescents: a preliminary study. Eur Rev Med Pharmaco/ Sci 2014;18:303-10.

17 Ho C-S, Huang J-Y, Yang C-H, et al. Is the Yale global tic severity scale a valid tool for parent-reported assessment in the paediatric population? A prospective observational study in Taiwan. BMJ Open 2020;10:e034634.
18 Kircanski K, Woods DW, Chang SW, et al. Cluster analysis of the Yale global tic severity scale (YGTSS): symptom dimensions and clinical correlates in an outpatient youth sample. J Abnorm Child Psychol 2010:38:777-88.

19 Campbell DE, Mehr S. Fifty years of allergy: 1965-2015. J Paediatr Child Health 2015;51:91-3.

20 Ryan MW, Marple BF, Leatherman B, et al. Current practice trends in allergy: results of a United States survey of otolaryngologists, allergistimmunologists, and primary care physicians. Int Forum Allergy Rhinol 2014:4:789-95. :

21 Catal F, Topal E, Soylu N, et al. Psychiatric disorders and symptoms severity in preschool children with atopic eczema. Allergol Immunopathol 2016:44:120-4.

22 Chen M-H, Su T-P, Chen Y-S, et al. Attention deficit hyperactivity disorder, tic disorder, and allergy: is there a link? A nationwide population-based study. J Child Psychol Psychiatry 2013;54:545-51.

23 Ludlow AK, Rogers SL. Understanding the impact of diet and nutrition on symptoms of Tourette syndrome: a scoping review. J Child Health Care 2018;22:68-83.

24 Bellanti JA, Settipane RA. Asthma, allergy, and psychiatric disease. Allergy Asthma Proc 2015;36:415-7.

25 Chang Y-T, Li Y-F, Muo C-H, et al. Correlation of Tourette syndrome and allergic disease: nationwide population-based case-control study. J Dev Behav Pediatr 2011:32:98-102.

26 Zhou H, Chen Z, Zhao W, et al. Evaluation of neuropsychiatric comorbidities and their clinical characteristics in Chinese children with asthma using the mini kid tool. BMC Pediatr 2019;19:454.

27 Yang J, Hirsch L, Osland S, et al. Health status, health related behaviours and chronic health indicators in people with Tourette syndrome: a Canadian population-based study. Psychiatry Res 2017;250:228-33.

28 Tagwerker Gloor F, Walitza S, Disorders T. Tic disorders and Tourette syndrome: current concepts of etiology and treatment in children and adolescents. Neuropediatrics 2016;47:84-96.

29 Samsam M, Ahangari R, Naser SA. Pathophysiology of autism spectrum disorders: revisiting gastrointestinal involvement and immune imbalance. World J Gastroenterol 2014;20:9942-51.

30 Li E, Ruan Y, Chen Q, et al. Streptococcal infection and immune response in children with Tourette's syndrome. Childs Nerv Syst 2015;31:1157-63.

31 McGuire JF, Hanks C, Lewin AB, et al. Social deficits in children with chronic tic disorders: phenomenology, clinical correlates and quality of life. Compr Psychiatry 2013;54:1023-31.

32 Spinello C, Laviola G, Macrì S. Pediatric Autoimmune Disorders Associated with Streptococcal Infections and Tourette's Syndrome in Preclinical Studies. Front Neurosci 2016;10:310.

33 Elamin I, Edwards MJ, Martino D. Immune dysfunction in Tourette syndrome. Behav Neurol 2013:27:23-32.

34 Ganor Y, Levite M. The neurotransmitter glutamate and human T cells: glutamate receptors and glutamate-induced direct and potent effects on normal human T cells, cancerous human leukemia and lymphoma T cells, and autoimmune human T cells. J Neural Transm 2014;121:983-1006.

35 Wang H-C, Lau C-I, Lin C-C, et al. Group A streptococcal infections are associated with increased risk of pediatric neuropsychiatric disorders: a Taiwanese population-based cohort study. J Clin Psychiatry 2016;77:e848-54.

36 Jeon S, Walkup JT, Woods DW, et al. Detecting a clinically meaningful change in tic severity in Tourette syndrome: a comparison of three methods. Contemp Clin Trials 2013:36:414-20. 